\title{
Region Tracking on Surfaces Deforming via Level-Sets Methods`
}

\author{
Marcelo Bertalmio $^{1}$, Guillermo Sapiro ${ }^{1}$, and Gregory Randall ${ }^{2}$ \\ 1 Electrical and Computer Engineering \\ University of Minnesota \\ Minneapolis, MN 55455 \\ guille@ece.umn.edu \\ 2 I.I.E. Facultad de Ingenieria \\ Universidad de la Republica \\ Montevideo, Uruguay
}

\begin{abstract}
Since the work by Osher and Sethian on level-sets algorithms for numerical shape evolutions, this technique has been used for a large number of applications in numerous fields. In medical imaging, this numerical technique has been successfully used for example in segmentation and cortex unfolding algorithms. The migration from a Lagrangian implementation to an Eulerian one via implicit representations or level-sets brought some of the main advantages of the technique, mainly, topology independence and stability. This migration means also that the evolution is parametrization free, and therefore we do not know exactly how each part of the shape is deforming, and the point-wise correspondence is lost. In this note we present a technique to numerically track regions on surfaces that are being deformed using the level-sets method. The basic idea is to represent the region of interest as the intersection of two implicit surfaces, and then track its deformation from the deformation of these surfaces. This technique then solves one of the main shortcomings of the very useful level-sets approach. Applications include lesion localization in medical images, region tracking in functional MRI visualization, and geometric surface mapping.
\end{abstract}

Key words: Level-sets, region tracking and correspondence, medical imaging, segmentation, visualization, shape deformation.

\section{Introduction}

The use of level-sets for the numerical implementations of $n$-dimensiona 11 shape deformations became extremely popular following the seminal work of Osher and Sethian [17] (see for example 1418 for some of the applications of this technique and a long list of references). In medical imaging, the technique has been successfully used for example for 2D and 3D segmentation [5:10 12 15 20]21].

\footnotetext{
${ }^{1}$ In this note we consider $n \geq 3$.
} 
The basic idea is to represent the deformation of an $n$-dimensional closed surface $\mathcal{S}$ as the deformation of an $n+1$-dimensional function $\Phi$. The surface is represented in an implicit form in $\Phi$, for example, via its zero level-set. Formally, let's represent the initial surface $\mathcal{S}(0)$ as the zero level-set of $\Phi$, i.e., $\mathcal{S}(0) \equiv\left\{\mathbf{X} \in \mathbb{R}^{n}: \Phi(\mathbf{X}, 0)=0\right\}$. If the surface is deforming according to

$$
\frac{\partial \mathcal{S}(t)}{\partial t}=\beta \mathcal{N}_{\mathcal{S}},
$$

where $\mathcal{N}_{\mathcal{S}}$ is the unit normal to the surface, then this deformation is represented as the zero level-set of $\Phi(\mathbf{X}, t): \mathbb{R}^{n} \times[0, \tau) \rightarrow \mathbb{R}$ deforming according to

$$
\frac{\partial \Phi(\mathbf{X}, t)}{\partial t}=\beta(\mathbf{X}, t)\|\nabla \Phi(\mathbf{X}, t)\|,
$$

where $\beta(\mathbf{X}, t)$ is computed on the level-sets of $\Phi(\mathbf{X}, t)$. The formal analysis of this algorithm can be found for example in 677.

The basic idea behind this technique is that we migrate from a Lagrangian implementation (particles on the surface) to an Eulerian one, i.e., a fix Cartesian coordinate system. This allows for example to automatically follow changes in the topology of the deforming surface $\mathcal{S}$, since the topology of the function $\Phi$ is fixed. See the mentioned references for more details on the level-sets technique.

In a number of applications, it is important not just to know how the whole surface deforms, but also how some of its regions do. Since the parametrization is missing, this is not possible in a straightforward level-sets approach. This problem is related to the aperture problem in optical flow computation, and it is also the reason why the level-sets approach can only deal with parametrization independent flows that do not contain tangential velocities. Although tangential velocities do not affect the geometry of the deforming shape, they do affect the 'point correspondence' in the deformation. For example, with a straight levelsets approach, it is not possible to determine where a given point $\mathbf{X}_{0} \in \mathcal{S}(0)$ is at certain time $t$. One way to solve this problem is to track isolated points with a set of ODE's, and this was done for example in grid generation and surface flattening; see 918 . This is a possible solution if we are just interested in tracking a number of isolated points. If we want to track regions for example, then using 'particles' brings us back to a 'Lagrangian formulation' and some of the problems that actually motivated the level-sets approach. For example, what happens if the region splits during the deformation? What happens if the region of interest is represented by particles that start to come too close together in some parts of the region and too far from each other in others?

In this note we propose an alternative solution to the problem of region tracking on surface deformations implemented via level-sets.2 The basic idea is to represent the boundary of the region of interest $\mathcal{R} \in \mathcal{S}$ as the intersection

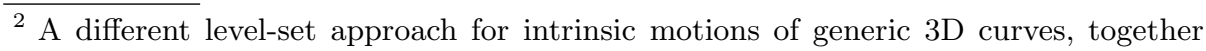
with very deep and elegant theoretical results, is introduced in [1. This approach is difficult to implement numerically, and in some cases not fully appropriate for numerical 3D curve evolution [16. A variation of this technique, with very good 
of the given surface $\mathcal{S}$ and an auxiliary surface $\hat{\mathcal{S}}$, both of them given as zero level-sets of $n+1$-dimensional functions $\Phi$ and $\hat{\Phi}$ respectively. ${ }^{3}$ The tracking of the region $\mathcal{R}$ is given by tracking the intersection of these two surfaces, that is, by the intersection of the level-sets of $\Phi$ and $\hat{\Phi}$. In the rest of this note we give details on the technique and present examples.

Note that although we use the proposed technique to track regions of interest on deforming surfaces, with the region deformation dictated by the surface deformation, the same general approach here presented of simultaneously deforming $n$ hypersurfaces $(n \geq 2)$ and looking at the intersection of their level-sets can be used for the numerical implementation of generic geometric deformations of curves and surfaces of high co-dimension 4

\section{The algorithm}

Assume the deformation of the surface $\mathcal{S}$, given by (1), is implemented using the level-sets algorithm, i.e., Equation (2). Let $\mathcal{R} \in \mathcal{S}$ be a region we want to track during this deformation, and $\partial \mathcal{R}$ its boundary. Define a new function $\hat{\Phi}(\mathbf{X}, 0): \mathbb{R}^{n} \rightarrow \mathbb{R}$ (a distance function for example), such that the intersection of its zero level-set $\hat{\mathcal{S}}$ with $\mathcal{S}$ defines $\partial \mathcal{R}$ and then $\mathcal{R}$. In other words,

$$
\partial \mathcal{R}(0):=\mathcal{S}(0) \cap \hat{\mathcal{S}}(0)=\left\{\mathbf{X} \in \mathbb{R}^{n}: \Phi(\mathbf{X}, 0)=\hat{\Phi}(\mathbf{X}, 0)=0\right\}
$$

The tracking of $\mathcal{R}$ is done by simultaneously deforming $\Phi$ and $\hat{\Phi}$. The auxiliary function $\hat{\Phi}$ deforms according to

$$
\frac{\partial \hat{\Phi}(\mathbf{X}, t)}{\partial t}=\hat{\beta}(\mathbf{X}, t)\|\nabla \hat{\Phi}(\mathbf{X}, t)\|,
$$

and then $\hat{\mathcal{S}}$ deforms according to

$$
\frac{\partial \hat{\mathcal{S}}}{\partial t}=\hat{\beta} \mathcal{N}_{\hat{\mathcal{S}}}
$$

We have then to find the velocity $\hat{\beta}$ as a function of $\beta$. In order to track the region of interest, $\partial \mathcal{R}$ must have exactly the same geometric velocity both in (2) and (3). The velocity in (2) (or (1)) is given by the problem in hand, and is

experimental results, is introduced in [11]. The Ambrosio-Soner approach and its variations deal with intrinsic curve-velocities and do not address the surface-velocity projection needed for the tracking in this paper.

3 The use of multiple level-set functions was used in the past for problems like motion of junctions [13. Both the problem and its solution are different from the ones in this paper.

${ }^{4}$ After this paper was accepted for publication, we became aware of recent work by Osher and colleagues using this general approach mainly to deform curves in $3 \mathrm{D}$ and curves on surfaces [4]. This work also does not deal with the projection of velocities as needed for our application. 
$\beta \mathcal{N}_{\mathcal{S}}$. Therefore, the velocity in (44) will be the projection of this velocity into the normal direction $\mathcal{N}_{\hat{\mathcal{S}}}$ (recall that the tangential component of the velocity does not affect the geometry of the flow). That is, for (at least) $\partial \mathcal{R}$,

$$
\hat{\beta}=\beta \mathcal{N}_{\mathcal{S}} \cdot \mathcal{N}_{\hat{\mathcal{S}}} .
$$

Outside of the region corresponding to $\mathcal{R}$, the velocity $\hat{\beta}$ can be any function that connects smoothly with the values in $\partial \mathcal{R} 5$

This technique, for the moment, requires to find the intersection of the zerolevel sets of $\Phi$ and $\hat{\Phi}$ at every time step, in order to compute $\hat{\beta}$. To avoid this, we choose a particular extension of $\hat{\beta}$ outside of $\partial \mathcal{R}$, and simple define $\hat{\beta}$ as the projection of $\beta \mathcal{N}_{\mathcal{S}}$ for all the values of $\mathbf{X}$ in the domain of $\Phi$ and $\hat{\Phi}$. 6 Therefore, the auxiliary level-sets flow is given by

$$
\begin{aligned}
\frac{\partial \hat{\Phi}}{\partial t}(\mathbf{X}, t) & =\left(\beta(\mathbf{X}, t) \frac{\nabla \Phi(\mathbf{X}, t)}{\|\nabla \Phi(\mathbf{X}, t)\|} \cdot \frac{\nabla \hat{\Phi}(\mathbf{X}, t)}{\|\nabla \hat{\Phi}(\mathbf{X}, t)\|}\right) \\
& \|\nabla \hat{\Phi}(\mathbf{X}, t)\|,
\end{aligned}
$$

and the region of interest $\mathcal{R}(t)$ is given by the portion of the zero level-sets that belongs to $\Phi(\mathbf{X}, t) \cap \hat{\Phi}(\mathbf{X}, t)$ :

$$
\partial \mathcal{R}(t)=\left\{\mathbf{X} \in \mathbb{R}^{n}: \Phi(\mathbf{X}, t)=\hat{\Phi}(\mathbf{X}, t)=0\right\} .
$$

For a number of velocities $\beta$, short term existence of the solutions to the levelsets flow for $\hat{\Phi}$ (in the viscosity framework) can be obtained from the results of Evans and Spruck 8 .

This formulation gives the basic region tracking algorithm. In the next section, we present some examples.

\section{Examples and comments}

We now present examples of the proposed technique. We should note that: (a) The numerical implementation of both the flows for $\Phi$ and $\hat{\Phi}$ follow the ordinary level-sets implementations developed by Osher and Sethian [17]; (b) Recently introduced fast techniques like narrow bands, fast-marching [18], or local methods [14, can also be used with the technique here proposed to evolve each one of the surfaces; (c) In the examples below, we compute a zero-order type of intersection between the implicit surfaces, meaning that we consider part of the intersection the full vortex where both surfaces go through (giving a jagged boundary). More

\footnotetext{
${ }^{5}$ To avoid the creation of spurious intersections during the deformation of $\Phi$ and $\hat{\Phi}$, these functions can be re-initialized every few steps, as frequently done in the level-sets approach.

${ }^{6}$ Note that although $\mathcal{S}$ and $\hat{\mathcal{S}}$ do not occupy the same regions in the $n$ dimensional space, their corresponding embedding functions $\Phi$ and $\hat{\Phi}$ do have the same domain, making this velocity extension straightforward.
} 
accurate intersections can be easily computed using sub-divisions as in marching cubes. Recapping, the same numerical implementations used for the classical level-sets approaches are used to implement the deformation of $\hat{\Phi}$, and finding the intersection is straightforward from algorithms like marching cubes.

Four examples are given in Figure 1 and Figure 2, one per column. In each example, the first figure on the top shows the original surface with the marked regions to be tracked (brighter regions), followed by three different time steps of the geometric deformation and region tracking.

Figure 1 shows two toy examples. We track the painted regions on the surfaces while they are deforming with a morphing type velocity $[23] .(\beta(\mathbf{X}, t)$ is simply the difference between the current surface $\Phi(\mathbf{X}, t)$ and a desired goal surface $\Phi(\mathbf{X}, \infty)$, two separate surfaces and two merged balls respectively, thereby morphing the initial surface toward the desired one [3].) Note how the region of interest changes topology (splits on the left example and merges on the next one).

Next, Figure 2 presents one of the main applications of this technique. Both these examples first show, on the top, a portion of the human cortex (whitematter/gray-matter boundary), obtained from MRI and segmented with the technique described in [19. In order to visualize brain activities recorder via functional MRI in one of the non-visible folds (sulci), it is necessary to 'unfold' the surface, while tracking the color-coded regions (surface unfolding or flattening has a number of applications in 3D medical imaging beyond fMRI visualization; see also 9]). In the first of these two examples (left column), the different gray values simply indicate sign of Gaussian curvature on the original surface (roughly indicating the sulci), while two arbitrary regions are marked in the last example (one of them with a big portion hidden inside the fold). We track each one of the colored regions with the technique described in this note. In the first column, $\beta(\mathbf{X}, t)=\frac{\operatorname{sign}\left(\kappa_{1}\right)+\operatorname{sign}\left(\kappa_{2}\right)}{2} \min \left(\left|\kappa_{1}\right|,\left|\kappa_{2}\right|\right)$, where $\kappa_{1}$ and $\kappa_{2}$ are the principal curvatures. In the second column, we use a morphing type velocity like before [2,3] (in this case, the desired destination shape is a convex surface). See [9] for additional possible unfolding velocities, including volume and area preserving ones. The colors on the deforming surfaces then indicate, respectively, the sign of the Gaussian curvature and the two marked regions in the original surfaces. Note how the surface is unfolded, hidden regions are made visible, and the tracking of the colored coded regions allow to find the matching places in the original 3D surface representing the cortex. This also allows for example to quantify, per each single tracked region, possible area/length distortions introduced by the flattening process. In order to track all the marked regions simultaneously in these two examples, we select the zero level-set of $\hat{\Phi}$ to intersect the zero level-set of $\Phi$ at all these regions. If we have regions with more than two color codes to track, as will frequently happen in fMRI, we just use one auxiliary function $\hat{\Phi}$ per color (region).

The same technique can be applied to visualize lesions that occur on the 'hidden' parts of the cortex. After unfolding, the regions become visible, and the region tracking allows to find their position in the original surface. When using 

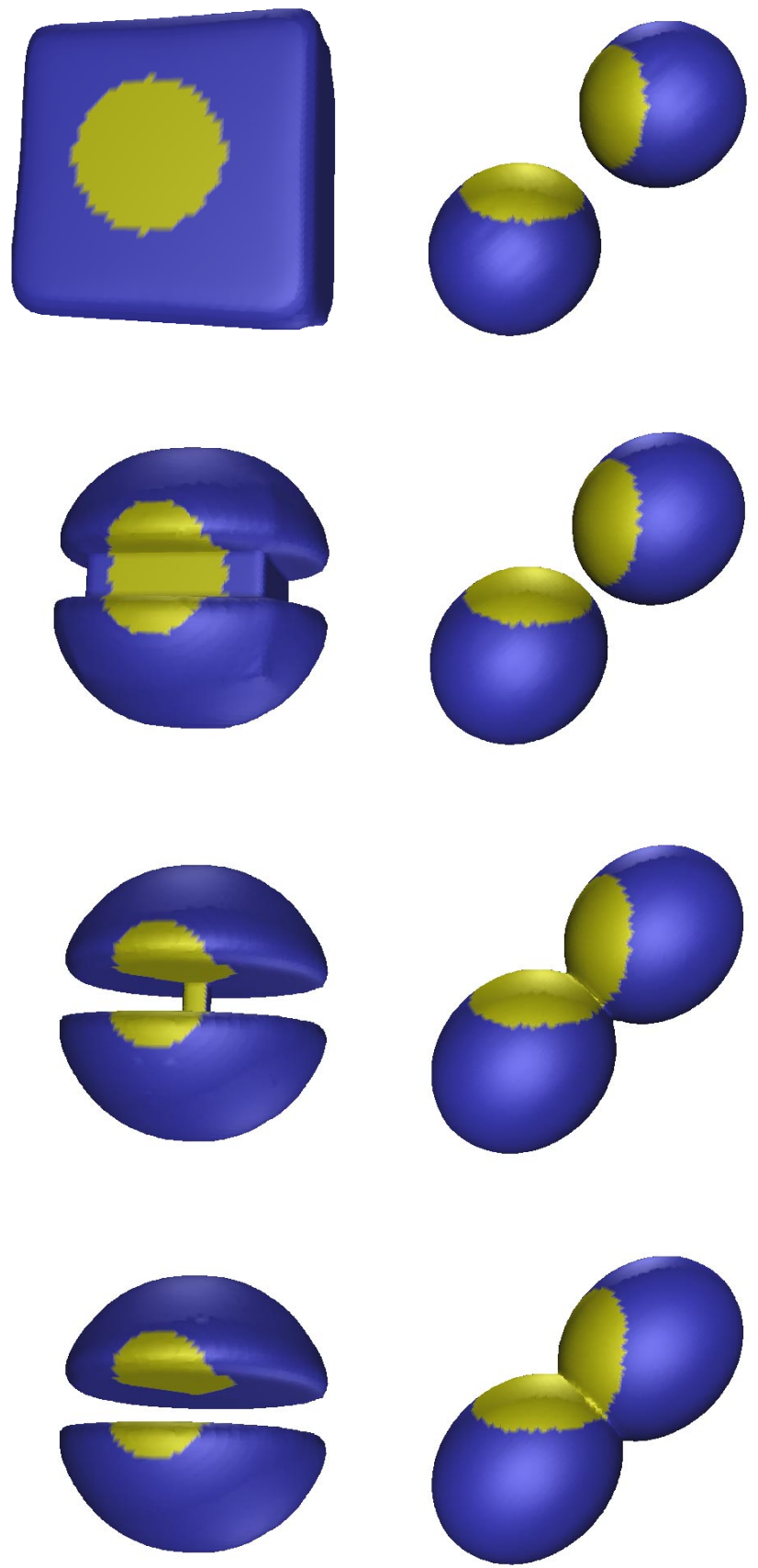

Fig. 1. Two simple examples, one per column, of the algorithm introduced in this note (brighter regions are the ones being tracked), demonstrating possible topological changes on the tracked region. 

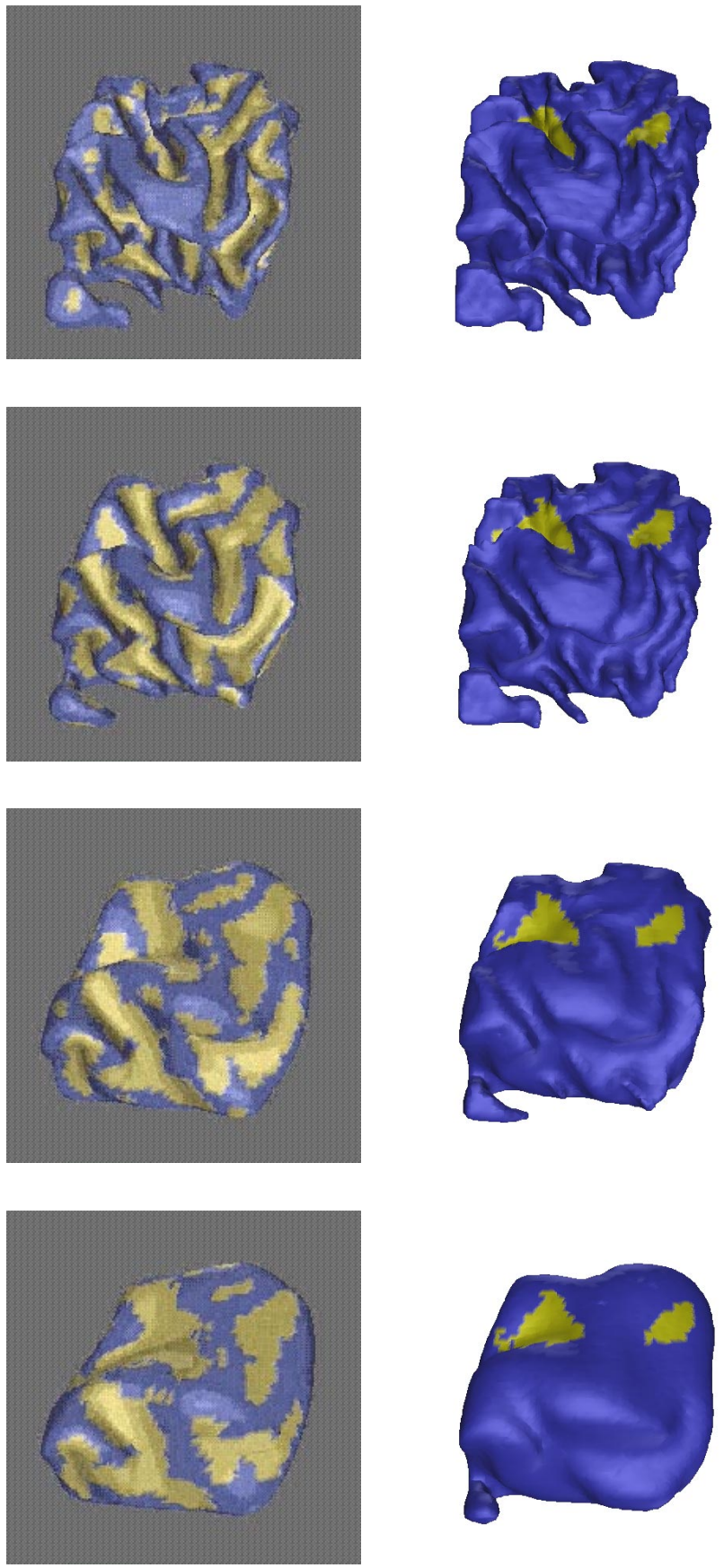

Fig. 2. Unfolding the cortex, and tracking the marked regions, with a curvature based flow and a $3 D$ morphing one, left and right columns respectively. 
level-sets techniques to deform two given shapes, one toward the other (a 3D cortex to a canonical cortex for example), this technique can be used to find the region-to-region correspondence. This technique then solves one of the basic shortcomings of the very useful level-sets approach.

\section{Acknowledgments}

GS wants to thank Prof. Brian Wandell from Stanford University for introducing him to the fMRI world and its unfolding issues. This was the motivation behind the algorithm described in this note. MB wants to thank the I.I.E. and the Facultad de Ingenieria, Montevideo, Uruguay for their continuous support through the M.Sc. Program and M.Sc. Scholarships. We thank Prof. Stanley Osher from UCLA and Prof. Olivier Faugeras from INRIA and MIT for making us aware of their related work. Comments from the anonymous reviewers are truly appreciated. This work was partially supported by NSF-LIS, by the Math, Computer, and Information Sciences Division at ONR, by an ONR Young Investigator Award, by the Presidential Early Career Awards for Scientists and Engineers (PECASE), by a National Science Foundation CAREER Award, by CSIC, and by CONICYT.

\section{References}

1. L. Ambrosio and M. Soner, "Level set approach to mean curvature flow in arbitrary codimension," Journal of Differential Geometry 43, pp. 693-737, 1996.

2. M. Bertalmio, Morphing Active Contours, M.Sc. Thesis, I.I.E., Universidad de la Republica, Uruguay, June 1998.

3. M. Bertalmio, G. Sapiro, and G. Randall, "Morphing active contours," Proc. IEEEICIP, Chicago, October 1998.

4. P. Burchard, L. T. Cheng, B. Merriman, and S. Osher, "Level set based motion of curves in $\mathbb{R}^{3}$," UCLA CAM Report, May 1999.

5. V. Caselles, R. Kimmel, and G. Sapiro, "Geodesic active contours," International Journal of Computer Vision 22:1, pp. 61-79, 19

6. Y. G. Chen, Y. Giga, and S. Goto, "Uniqueness and existence of viscosity solutions of generalized mean curvature flow equations," Journal of Differential Geometry 33, 1991.

7. L. C. Evans and J. Spruck, "Motion of level sets by mean curvature, I," Journal of Differential Geometry 33, 1991.

8. L. C. Evans and J. Spruck, "Motion of level sets by mean curvature, II," Trans. Amer. Math. Soc. 330, pp. 321-332, 1992.

9. G. Hermosillo, O. Faugeras, and J. Gomes, "Cortex unfolding using level set methods," INRIA Sophia Antipolis Technical Report 3663, April 1999.

10. L. M. Lorigo, O. Faugeras, W. E. L. Grimson, R. Keriven, and R. Kikinis, "Segmentation of bone in clinical knee MRI using texture-based geodesic active contours," Proceedings Medical Image Computing and Computer-Assisted Intervention, MICCAI '98, pp. 1195-1204, Cambridge, MA, Springer, 1998.

11. L. Lorigo, O. Faugeras, W.E.L. Grimson, R. Keriven, R. Kikinis, and C-F. Westin, "Co-dimension 2 geodesic active contours for MRA segmentation," International Conference on Information Processing in Medical Imaging, June 1999, forthcoming. 
12. R. Malladi, J. A. Sethian and B. C. Vemuri, "Shape modeling with front propagation: A level set approach," IEEE Trans. on PAMI 17, pp. 158-175, 1995.

13. B. Merriman, J. Bence, and S. Osher, "Motion of multiple junctions: A level-set approach,' Journal of Computational Physics 112, pp. 334-363, 1994.

14. B. Merriman, R. Caflisch, and S. Osher, "Level set methods, with an application to modeling the growth of thin films, UCLA CAM Report 98-10, February 1998.

15. W. J. Niessen, B. M. Romeny, and M. A. Viergever, "Geodesic deformable models for medical image analysis," IEEE Trans. Medical Imaging 17, pp. 634-641, 1998.

16. S. Osher, Personal communication, March 1999.

17. S. Osher and J. Sethian, "Fronts propagating with curvature-dependent speed: Algorithms based on Hamilton-Jacobi formulations," Journal of Computational Physics 79, pp. 12-49, 1988.

18. J. Sethian, Level Set Methods: Evolving Interfaces in Geometry, Fluid Mechanics, Computer Vision and Material Sciences, Cambridge University Press, Cambridge, UK, 1996.

19. P. Teo, G. Sapiro, and B. Wandell, "Creating connected representations of cortical gray matter for functional MRI visualization," IEEE Trans. Medical Imaging 16:06, pp. 852-863, 1997.

20. A. Yezzi, S. Kichenassamy, P. Olver, and A. Tannenbaum, "Geometric active contours for segmentation of medical imagery," IEEE Trans. Medical Imaging 16, pp. 199-210, 1997.

21. X. Zeng, L. H. Staib, R. T. Schultz, and J. S. Duncan, "Segmentation and measurement of the cortex from 3D MR Images," Proceedings Medical Image Computing and Computer-Assisted Intervention, MICCAI '98, pp. 519-530, Cambridge, MA, Springer, 1998. 